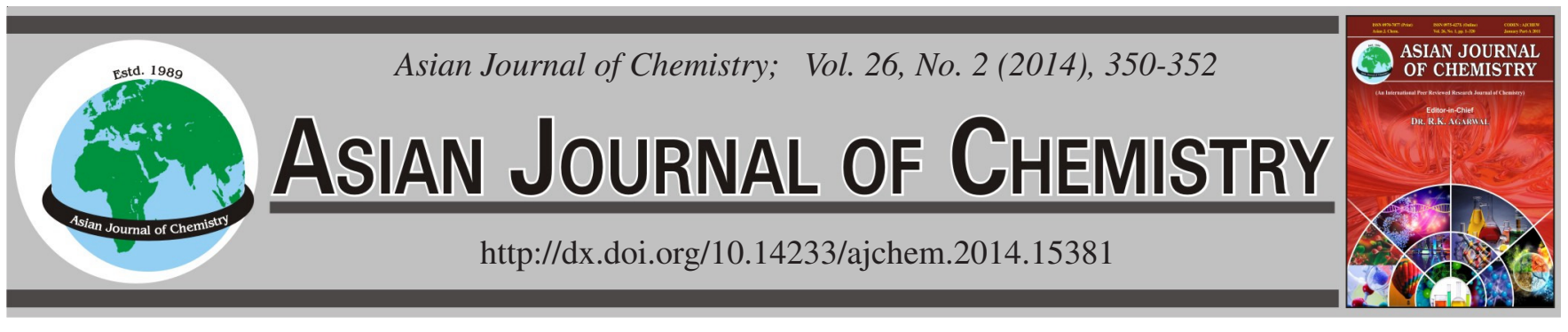

\title{
Synthesis and Characterization of Novel UV-Curable Waterborne Fluorinated Polyurethane Acrylate Latex
}

\author{
Hongbo Liang ${ }^{1,2, *}$, Cheng Lv ${ }^{1}$, Lei Xiong ${ }^{1, *}$, Shengmei Huang ${ }^{1}$ and HongQing Fan ${ }^{1}$
}

${ }^{1}$ School of Material Science and Engineering, Nanchang Hangkong University, Nanchang 330063, P.R. China

${ }^{2}$ State Key Laboratory of Polymer Materials Engineering (Sichuan University), Chendu 610065, P.R. China

*Corresponding authors: Tel: +86 791 83863025; E-mail: liahongbo@163.com; x_lei81@163.com

\begin{abstract}
In this study, a novel UV-curable waterborne fluorinated polyurethane acrylate (FPUA) latex was obtained from urethane acrylate with fluorinated acrylate copolymer prepared by free radical copolymerization. FT-IR analysis demonstrated that the fluorinated acrylate monomer had been introduced into the chain of the fluorinated polyurethane acrylate. XPS analysis showed that the atomic percent of fluorine on the air-film interface of fluorinated polyurethane acrylate films was much higher than that at the film-glass interface and this feature became more evident after heat treatment. In addition to, the effect of fluorinated monomers content on properties of the films was studied. The results showed that the hydrophobic/oleophobic properties and abrasion resistance of films were enhanced with the increase of fluorinated monomer content.
\end{abstract}

Keywords: UV-Curable, Polyurethane acrylic, Fluorinated, Hydrophobic/oleophobic properties, Abrasion resistance.

\section{INTRODUCTION}

In the past decade, ultraviolet-curable waterborne coatings have drawn significant attention in academics and industry for its unique combination of environmental protection, energy saving and selectivity and flexibility in the use ${ }^{1,2}$. Polyurethane (PU) has been popularly used as binders in UV-curable waterborne coatings due to its overall balance of properties. However, the water resistance and weatherability of polyurethane are poor because of the presence of the hydrophilic groups such as carboxyl groups in the molecule chains ${ }^{3}$. Compared to the polyurethane, polyacrylate (PA) type materials show excellent properties in terms of weatherability, water and solvent resistance. Therefore, it is a common practice to combine polyurethane with polyacrylate to improve the properties of the resulting materials ${ }^{4-7}$.

It is well known that the fluorinated groups are attractive that provide polymer with various interesting properties, such as low surface energy, hydrophobic/oleophobic ability, biocompatibility and excellent thermal stability ${ }^{8-10}$. The coatings based on polyurethane acrylate with fluorinated groups can exhibit several virtues of polyurethane acrylate and fluorinated polymer, which find wide application in aircraft, marine and biomedicine ${ }^{11}$.

In this paper, a novel UV-curable waterborne fluorinated polyurethane acrylate latex was synthesized based on isophorone diisocyanate (IPDI), 2-hydroxyethyl acrylate (HEA), methyl methacrylate (MMA), acrylic acid (AA), butyl acrylate (BA) and 2,3,4,5,6,6,6-heptafluoro-3,5-bis(trifluoromethyl)hexyl acrylate (HFHA). Structure and properties of fluorinated polyurethane acrylate films were investigated by FT-IR, XPS, abrasion resistance test and contact angles measurement.

\section{EXPERIMENTAL}

Isophorone diisocyanate (IPDI) was provided by Huels AG Company in Germany. 2-Hydroxyethyl acrylate (HEA), methyl methacrylate (MMA), acrylate acid (AA), butyl acrylate (BA), triethyl amine (TEA), acetone, dibutyltin dilaurate (DBTDL) and azobisisobutyronitrile (AIBN) were supplied by Xilong Chemical Co., Ltd. 2-Hydrozy-2-methyl-1-phenylpropan-1-one (Darocur 1173) was supplied by Ciba Fine Chemical. 2,3,4,5,6,6,6-Heptafluoro-3,5-bis(trifluoromethyl) hexyl acrylate (HFHA) was purchased from Harbin Xeogia fluorine-silicon chemical company.

Synthesis of urethane acrylate (HEA-IPDI): A mixture of $11.11 \mathrm{~g}$ of isophorone diisocyanate and $16 \mathrm{~g}$ of acetone was added to a three-neck flask with a mechanical stirrer, isobaric funnel and thermometer. $5.8 \mathrm{~g}$ of 2-hydroxyethyl acrylate was then dropped in system in $2 \mathrm{~h}$. The reaction mixture was stirred at $0^{\circ} \mathrm{C}$ in ice/water bath for $40 \mathrm{~min}$. The reaction temperature was then increased to $35^{\circ} \mathrm{C}$, where the mixture was maintained for $3 \mathrm{~h}$. The reaction was followed by determining the residual isocyanate groups (-NCO) content using the 
standard di- $n$-butylamine back titration method until the value of -NCO reached to the theoretical value.

Copolymerization of fluorinated acrylate/acrylate: After dissolving $1.6 \mathrm{~g}$ AIBN and $50.0 \mathrm{~g}$ xylene in a four-neck flask, $50 \mathrm{~g}$ butyl acetate were added into the flask, the reaction was conducted at $80^{\circ} \mathrm{C}$. The mixture of MMA, BA, AA, HEA and HFHA was added slowly into the flask in $3 \mathrm{~h}$. After the addition was completed, the reaction stirred at $80{ }^{\circ} \mathrm{C}$ for $6 \mathrm{~h}$. After cooling to room temperature, the resulted copolymer was washed with petroleum ether and acetone in turn to purify copolymer.

Preparation of fluorinated polyurethane acrylic (FPUA) latex: Typically, the resulted copolymer was dissolved by the acetone in a four-neck flask. Then a mixture of HEAIPDI with 2 wt $\%$ DBTDL and 5 wt $\% p$-hydroxyanisole was added dropwise over $2 \mathrm{~h}$ and the mixture was stirred at $50^{\circ} \mathrm{C}$ in oil bath for $3 \mathrm{~h}$. Triethylamine was added in the system as a neutralization agent under high speed shearing (1200 r/min) and then some deionized water was added to emulsify the system. Then UV-curable waterborne FPUA latex was obtained. The whole synthesis process of UV-curable waterborne FPUA is shown in the Fig. 1.
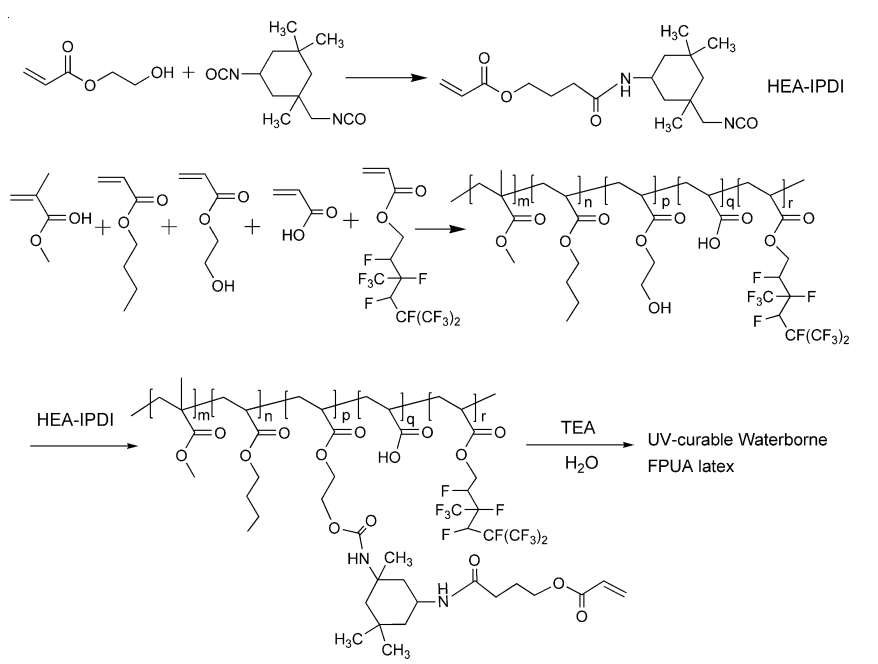

Fig. 1. Synthesis of UV-curable waterborne FPUA latex

Preparation of UV-cured films: The UV-cured FPUA films were formed by casting the formulations with $1.5 \mathrm{wt} \%$ photoinitiator Darocur 1173 onto a glass plate at room temperature. The films were then dried in an oven at $50{ }^{\circ} \mathrm{C}$ until reaching a constant weight. After that, the dry FPUA films were exposed to a medium pressure mercury lamp.

Measurements: Fourier transform infrared (FT-IR) spectra of the materials were recorded using a Bruker 210 IR spectrometer. X-Ray photoelectron spectroscopy (XPS) was carried on a PHI5000C ESCA System with an $\mathrm{MgK}_{\alpha} \mathrm{X}$-ray source. The different liquids contact angles on films were obtained from an OCA20 (Data Physics) contact angle goniometer. The abrasion resistance of prepared films was measured with the Taber Abraser 5135.

\section{RESULTS AND DISCUSSION}

Analysis of chemical structures of FPUA latex: The FT-IR spectrum of FPUA is presented in Fig. 2. The charac-

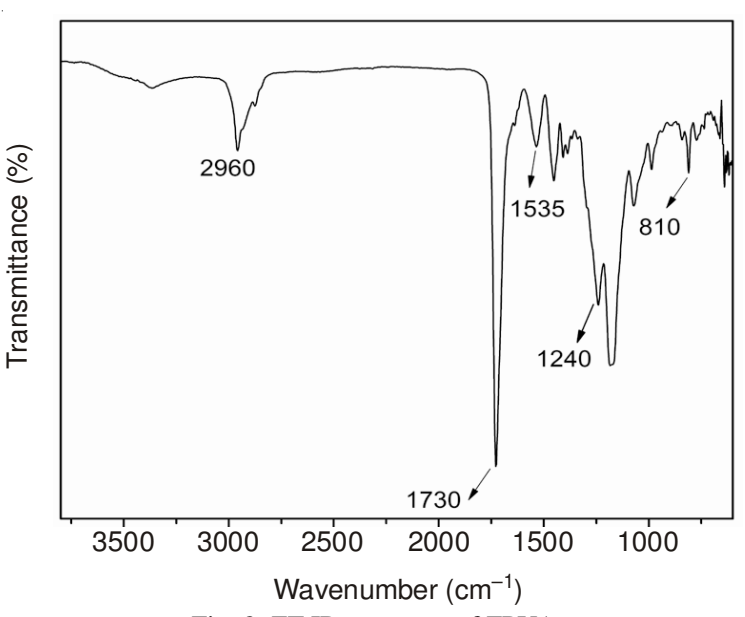

Fig. 2. FT-IR spectrum of FPUA

teristic absorption peak of -NCO groups at $2270 \mathrm{~cm}^{-1}$ can not be found in this spectrum and the vibrations of the $\mathrm{N}-\mathrm{H}$ groups occur at $1535 \mathrm{~cm}^{-1}$. These results indicate that all of the -NCO groups were reacted with -OH groups to form urethane linkages. The characteristic absorptions of $\mathrm{C}=\mathrm{C}$ at $810 \mathrm{~cm}^{-1}$ are observed. In addition to, the absorption peaks of $\mathrm{C}-\mathrm{H}$ and $\mathrm{C}=\mathrm{O}$ groups are strongly shown at $3000-2800$ and $1730 \mathrm{~cm}^{-1}$, respectively, resulting from BA, MMA, AA, HEA and HFHA units. The peaks at $1240 \mathrm{~cm}^{-1}$ originated from C-F are also found in this spectrum. Thus, FT-IR spectrum reveals that the HFHA monomers have been introduced into the copolymer.

Wettability of latex films: The surface wettability of UVcured films with various HFHA contents was studied by using a contact angle meter. Fig. 3 shows the contact angles of water and bromonaphthalene on the air-side surface of the films containing different content of HFHA. It can be found that the contact angles of water on the surface of films are increased with the increase of the HFHA content. As the HFHA content in the films is increased from $0-3 \mathrm{wt} \%$, the contact angles of water on the surface increase from 78.1-102.1 ${ }^{\circ}$. Then the increased tendency of the water contact angle begins to weaken and reaches its maximum value at $5 \mathrm{wt} \%$ HFHA. For the contact angles of bromonaphthalene on the surface of film, a similar trend can be observed. The bromonaphthalene contact angles increase from $28.1^{\circ}$ and reached a maximum value of $62.3^{\circ}$ with 5 wt $\%$ HFHA. According to the contact angle measurement, the fluorine-containing films show excellent water and oil repellence compared to the non-fluorine-containing films. This behaviour may be attributed to the presence of hydrophobic/oleophobic tail-like fluorine-substituted chains on the surfaces of UV-cured FPUA films.

XPS analysis of films: To study the water and oil repellency of the FPUA films with 5 wt \% HFHA, the content of various elements on the surface of films were investigated by XPS analysis. The XPS spectra of different interface of UVcured FPUA films are given in Fig. 4. The XPS spectra show the characteristic peaks of fluorine ( $\mathrm{F} 1 \mathrm{~s}$ at $688.6 \mathrm{eV})$, oxygen (O1s at $532.8 \mathrm{eV})$, carbon (C1s at $284.8 \mathrm{eV}$ ) and nitrogen $(\mathrm{N} 1 \mathrm{~s}$ at $399.6 \mathrm{eV})$. According XPS results, the atomic percent of fluorine at air-film interface $(12.35 \%)$ is much bigger than that at the film-glass interface $(5.18 \%)$ and the theoretically value $(3.3 \%)$ calculated by recipe. However, the atomic 


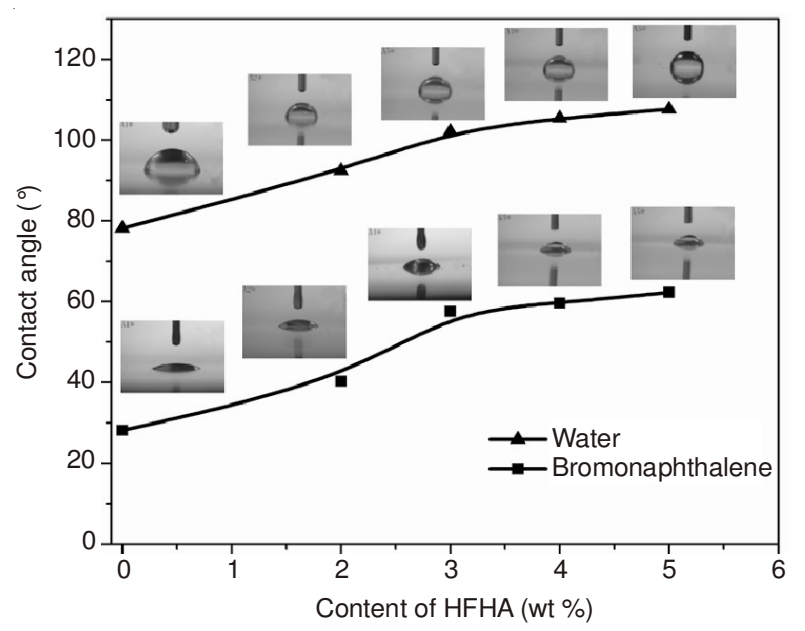

Fig. 3. Effect of HFHA content on contact angle of water and bromonaphthalene

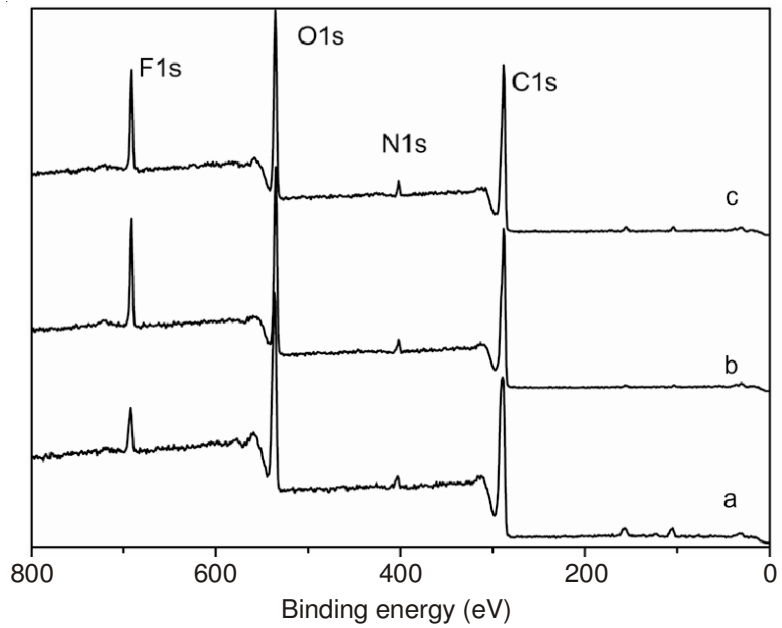

Fig. 4. XPS spectra of (a) film-glass interface, (b) air-film interface and (c) air-film interface after heat treatment

per cent of carbon at the film-glass interface is increased. This result indicates that the existence of an aggregation of fluorine on the air-film interface. In addition to, the aggregation of fluorine on the air-film interface becomes more evident after heat treatment. XPS analysis further demonstrates that the fluoroalkyl groups tend to migrate to air-film interface and occupy it during the film formation.

Abrasion resistance properties of films: The abrasion resistance is expressed by the mass loss calculated by weighing the samples every 25 abrasion cycles. The mass decrease caused by using $1000 \mathrm{~g}$ load and CS10 abrading grinders. Fig. 5 shows the effect of HFHA content on the abrasion resistance properties of films. As the specimens are abraded for 25 cycles, the mass loss of non-fluorine-containing film is much bigger than that of the fluorine-containing film. The mass loss of nonfluorine-containing is $c a .2 .1 \mathrm{mg}$ in 25 cycles, while the mass loss of film with $3 \mathrm{wt} \%$ HFHA is only ca. $0.3 \mathrm{mg}$. The increase of abrasion resistance of fluorine-containing film is attributed to the special properties of the fluoroalkyl groups, which have a tendency to migrate to the air-film interface and occupy the outmost surface. The presence of fluoroalkyl groups on the surface of films can form a cross-linked network containing $\mathrm{F}$

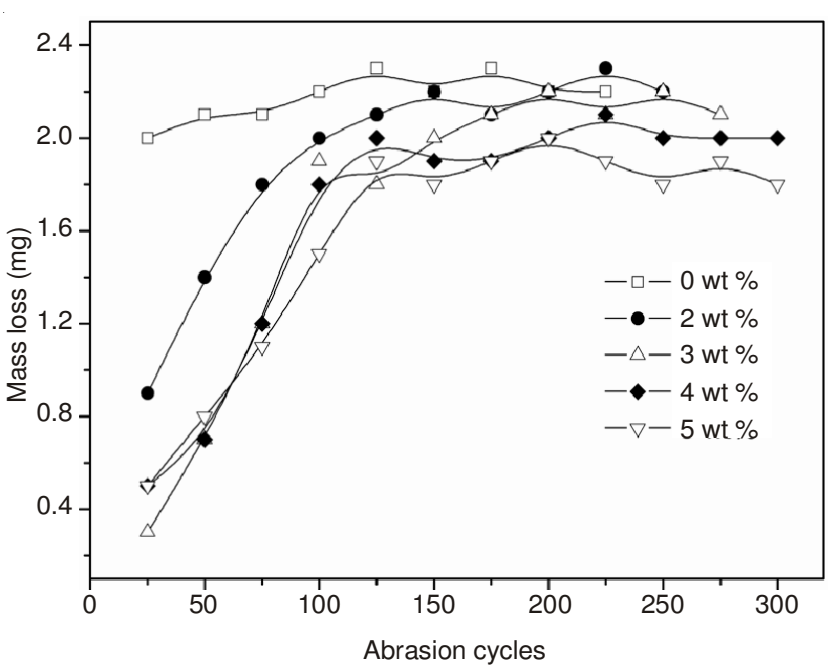

Fig. 5. Effect of HFHA content on abrasion resistance properties of films

atoms and increase the abrasion resistance of films. After 100 cycles, as the cross-linked network is destroyed, the mass loss of FPUA films increases significantly. However, it is still smaller than that of non-fluorine-containing film because of the presence of residual $\mathrm{F}$ atoms on the surface.

\section{Conclusion}

In this work, we prepared a novel UV-curable waterborne fluorinated polyurethane acrylate latex. FT-IR analysis demonstrated that the synthesis of FPUA was successful. XPS results showed that there was an aggregation of fluorine on the air-film interface, which became more evident after heat treatment. The incorporation of HFHA monomers can enhance the hydrophobic/oleophobic properties of films. Also the increase of HFHA content incorporated into the formulations can enhance the abrasion resistance of UV-cured films.

\section{ACKNOWLEDGEMENTS}

The authors are grateful for the financial support by National Natural Science Foundation of China (No. 51103069), Science Foundation of Aeronautics of China (No. 2011ZF56018), Natural Science Foundation of Jiangxi Province (No. 20122BAB216014) and the Opening Project of State Key Laboratory of Polymer Materials Engineering (Sichuan University) (No. KF201306).

\section{REFERENCES}

1. Y. Heischkel, K. Menzel, W. Paulus, C. Decker, R. Schwalm, Polym. Prep., 44, 9 (2003).

2. D.K. Chattopadhyay and K.V.S.N. Raju, Prog. Polym. Sci., 32, 352 (2007).

3. C. Zhang and S.Y. Feng, Polym. Int., 53, 1936 (2004).

4. X.Y. Xiao and R. Xu, J. Appl. Polym. Sci., 119, 1576 (2011).

5. T. Jeevananda and Siddaramaiah, Eur. Polym. J., 39, 569 (2003).

6. H. Tanaka, Y. Suzuki and F. Yoshino, Colloid. Surf. A, 153, 597 (1999).

7. H.J. Adler, K. Jahny and B. Vogt-Birnbrich, Prog. Org. Coat., 43, 251 (2001).

8. A.E. Feiring and E.R. Wonchoba, Macromolecules, 31, 7103 (1998).

9. I. Erol, J. Fluorine Chem., 129, 613 (2008).

10. G.C. Jiang, X.L. Tuo, D.R. Wang and Q. Li, J. Polym. Sci. A, 47, 3248 (2009).

11. B. Jones, J. Coat. Technol., 5, 44 (2008). 